\title{
Therapeutic Advances in Autism and Other Neurodevelopmental Disorders
}

\author{
Jeffrey L. Neul ${ }^{1} \cdot$ Mustafa Sahin $^{2}$
}

Published online: 16 June 2015

(C) The American Society for Experimental NeuroTherapeutics, Inc. 2015

According to the Diagnostic and Statistical Manual of Mental Disorders, 5th Edition, autism spectrum disorder (ASD) is defined by persistent deficits in social communication and social interaction, as well as by restricted, repetitive patterns of behavior, interests, or activities. Recent advances in genetic research indicate that there may be nearly 1000 genes that contribute to the susceptibility of developing ASD. While the genetic revolution has enabled us to identify a genetic etiology in a significant proportion of our patients, the number of treatments available to treat patients with ASD has not changed. For example, while risperidone and aripiprazole have been approved by the US Food and Drug Administration for treating irritability associated with ASD, there are no drugs currently approved treating autism's 2 core characteristics - deficits in social communication/interaction, and restricted, repetitive patterns of behavior, interests, or activities. Behavioral treatments, such as applied behavior analysis, remain the mainstay of therapeutic options in ASD today. Because it has been 5 years since an issue of Neurotherapeutics was dedicated to ASD, we thought it was appropriate time for an update in the field.

This issue on ASD is made up of 2 sections. The first reviews the advances in techniques used to study ASD in the laboratory and in the clinic overall. The second contains reviews of individual rare diseases that are highly associated

Jeffrey L. Neul

jneul@ucsd.edu

$\checkmark$ Mustafa Sahin

Mustafa.Sahin@childrens.harvard.edu

1 Division of Child Neurology, Department of Neurosciences, University of California, San Diego, La Jolla, CA, USA

2 Department of Neurology, F.M. Kirby Neurobiology Center, Boston Children's Hospital, Harvard Medical School, Boston, MA, USA with ASD. Many of these diseases are single-gene defects or copy number variants and represent the best-investigated genetic causes of ASD. In most cases, we invited experts from the clinical and the basic science perspectives to join in preparing these updates. Thus, they represent a broad spectrum of translational efforts ongoing in ASD in 2015.

In the first section, Ellegood and Crawley [1] review the animal models of autism and their phenotyping, using behavioral assays, neuroanatomy and imaging. Acab and Muotri [2] discuss the promise and challenges of induced pluripotent stem cell (IPSC) technology in neurodevelopmental disorders. IPSCs have been developed for several genetically defined conditions. Neurons and other central nervous system cell types derived from IPSCs hold the promise of shedding light on the underlying pathophysiology, as well as serving as platforms for drug discovery. Levin and Nelson [3] discuss potential biomarkers with relevance to autism, with particular emphasis on inhibitory circuits.

It is becoming clear that ASD can be caused by monogenic or polygenic causes. Many of the genetic variants will be common variants with a small effect size, while others will be rare variants with larger effect size and higher penetrance. Obviously, this creates an enormous challenge in terms of developing treatments for these diverse genetic disorders. Therefore, much of the effort in developing treatment for neurodevelopmental disorders has focused on rare disorders with high penetrance of ASD. The second section starts with an overview by Gamsiz et al. [4] on rare genetic diseases that are highly penetrant for ASD, and includes reviews, each addressing a specific neurogenetic disease. Davis et al. [5] describe progress in tuberous sclerosis complex, a disease in which the mechanistic target of rapamycin pathway is hyperactive. Gross et al. [6] review the ups and downs of the journey in fragile $\mathrm{X}$ syndrome over the last decade. Tilot et al. [7] discuss advances in phosphatase and tensin homologue 
protein on chromosome 10-related macrocephaly and autism. Costales and Kolevzon [8] provide an update on PhelanMcDermid syndrome in which deficiency in a postsynaptic density protein, SHANK3, is associated with autism, intellectual disability, and epilepsy. Pozzo-Miller et al. [9] review how preclinical studies in methyl-CpG-binding protein 2deficient mice have led to clinical trials in Rett syndrome. Finally, Margolis et al. [10] present a clinical and genetic overview of Angelman syndrome and the recent efforts in mechanism-based therapies for this disorder.

We anticipate that this issue will reveal what an unprecedented time this is for ASD research. The advances in genetics and neurobiology have culminated in identification of molecular features common to a number of genetically distinct forms of ASD. These advances have led to a number of potential treatment targets in several genetic disorders associated with ASD. The preclinical studies in mice have been very promising; however, as yet, none has shown to be effective in clinical trials. In the meantime, we now recognize that there are several hundred genes that can contribute to the susceptibility for ASD. The dream is that some of the molecular features present and therapies that are proven to be effective in rare diseases such as fragile $\mathrm{X}$ syndrome, tuberous sclerosis complex, and Rett syndrome will also be effective in subsets of patients with "idiopathic" ASD. We suspect the next issue of Neurotherapeutics on ASD will contain some examples of such successes.

Acknowledgements J.L.N. and M.S. are both funded by the Rare Diseases Clinical Research Network (RDCRN) (U54HD061222 and U54NS092090, respectively). RDCRN is an initiative of the Office of
Rare Diseases Research (ORDR), National Center for Advancing Translational Sciences (NCATS), funded through collaboration between NCAT S, National Institute of Mental Health, National Institute of Neurological Disease and Stroke and National Institute of Child Health and Human Development.

Required Author Forms Disclosure forms provided by the authors are available with the online version of this article.

\section{References}

1. Ellegood J, Crawley JN. Behavioral and neuroanatomical phenotypes in mouse models of autism. Neurotherapeutics 2015.

2. Acab A, Muotri AR. The use of induced pluripotent stem cell technology to advance autism research and treatment. Neurotherapeutics 2015.

3. Levin AR, Nelson CA. Inhibition-based biomarkers for autism spectrum disorder. Neurotherapeutics 2015.

4. Gamsiz ED, Sciarra LN, Maguire AM, Pescosolido MF, van Dyck L, Morrow EM. Discovery of rare mutations in autism: elucidating neurodevelopmental mechanisms. Neurotherapeutics 2015.

5. Davis PE, Peters JM, Krueger DA, Sahin M. Tuberous sclerosis: a new frontier in targeted treatment of autism. Neurotherapeutics 2015.

6. Gross C, Hoffmann A, Bassell GJ, Berry-Kravis EM. Therapeutic strategies in fragile $\mathrm{X}$ syndrome: from bench to bedside and back. Neurotherapeutics 2015.

7. Tilot AK, Frazier TW, II, Eng C. Balancing proliferation and connectivity in $P T E N$-associated autism spectrum disorder. Neurotherapeutics 2015.

8. Costales JL, Kolevzon A. Phelan-McDermid syndrome and SHANK3: implications for treatment. Neurotherapeutics 2015.

9. Pozzo-Miller L, Pati S, Percy AK. Rett syndrome: reaching for clinical trials. Neurotherapeutics 2015.

10. Margolis SS, Sell GL, Zbinden MA, Bird LM. Angelman syndrome. Neurotherapeutics 2015. 